\title{
Current Opinion on Endovascular Therapy for Emergent Large Vessel Occlusion Due to Underlying Intracranial Atherosclerotic Stenosis
}

\author{
Dong-Hun Kang, $\mathrm{MD}^{1,2}$, Woong Yoon, $\mathrm{MD}, \mathrm{PhD}^{3}$ \\ Departments of ${ }^{1}$ Neurosurgery and ${ }^{2}$ Radiology, School of Medicine, Kyungpook National University, Daegu, Korea; ${ }^{3}$ Department of Radiology, \\ Chonnam National University Medical School, Gwangju, Korea
}

For recanalization of emergent large vessel occlusions (ELVOs), endovascular therapy (EVT) using newer devices, such as a stent retriever and large-bore catheter, has shown better patient outcomes compared with intravenous recombinant tissue plasminogen activator only. Intracranial atherosclerotic stenosis (ICAS) is a major cause of acute ischemic stroke, the incidence of which is rising worldwide. Thus, it is not rare to encounter underlying ICAS during EVT procedures, particularly in Asian countries. ELVO due to underlying ICAS is often related to EVT procedure failure or complications, which can lead to poor functional recovery. However, information regarding EVT for this type of stroke is lacking because large clinical trials have been largely based on Western populations. In this review, we discuss the unique pathologic basis of ELVO with underlying ICAS, which may complicate EVT procedures. Moreover, we review EVT data for patients with ELVO due to underlying ICAS and suggest an optimal endovascular recanalization strategy based on the existing literature. Finally, we present future perspectives on this subject.

Keywords: Atherosclerosis; Intracranial; Cerebral infarction; Endovascular; Thrombectomy; Glycoprotein-IIb/IIIa inhibitor; Percutaneous transluminal angioplasty; Stenting

\section{INTRODUCTION}

Over the past two decades, intravenous administration of recombinant tissue plasminogen activator has been the only standard therapy for the treatment of acute ischemic stroke despite its limited efficacy for recanalization of emergent large vessel occlusion (ELVO) (1-4). Recently, several randomized clinical trials on endovascular therapy (EVT) using a stent retriever have shown better treatment outcomes

Received November 22, 2018; accepted after revision January 20, 2019.

Corresponding author: Woong Yoon, MD, PhD, Department of Radiology, Chonnam National University Medical School, Chonnam National University Hospital, 42 Jebong-ro, Dong-gu, Gwangju 61469, Korea.

- Tel: (8262) 220-5746 - Fax: (8262) 226-4380

- E-mail: radyoon@jnu.ac.kr

This is an Open Access article distributed under the terms of the Creative Commons Attribution Non-Commercial License (https://creativecommons.org/licenses/by-nc/4.0) which permits unrestricted non-commercial use, distribution, and reproduction in any medium, provided the original work is properly cited. in patients with acute stroke due to ELVO compared with intravenous thrombolysis only (5-8). Of the various causes of ELVO, intracranial atherosclerotic stenosis (ICAS) has garnered little attention from treating physicians compared with other stroke etiologies, such as embolic occlusions. This is probably because previous randomized trials have mostly been conducted in Western populations (9-11).

ICAS is a progressive disease characterized by the accumulation of lipids and fibrous elements in the walls of intracranial arteries, which leads to arterial changes ranging from mild wall thickening to hemodynamically significant luminal stenosis $(12,13)$. ICAS can lead to acute ischemic stroke through various mechanisms, including in situ thrombotic occlusion, artery-to-artery embolism, hemodynamic compromise, and branch vessel occlusion. of these mechanisms, ELVO is typically related to in situ thrombotic occlusion, which occurs when unstable plaque ruptures in patients with advanced ICAS $(10,12)$. This type of ELVO is often associated with complicated or failed endovascular thrombectomy procedures, especially when the 
treating neurointerventionists have little knowledge about the characteristics and management of this special type of stroke $(14,15)$. In this review, we discuss the unique pathologic basis of ELVO with underlying ICAS, which may complicate EVT procedures. Additionally, we review EVT data for patients with ELVO due to underlying ICAS and suggest an optimal endovascular recanalization strategy based on the existing literature. Finally, we present future perspectives on this subject.

\section{Clinical Characteristics of Patients with ELVO Due to Underlying ICAS}

Worldwide, 20-40 per 100000 population are estimated to have experienced ICAS-related stroke. ICAS is more prevalent in Asian, Black, and Hispanic populations than in Western populations $(10,11)$. In one Korean study, ICAS with $>50 \%$ luminal stenosis was found in $29.6 \%$ of patients with acute infarction or transient ischemic stroke and nonvalvular atrial fibrillation (9). A Chinese postmortem study reported that $31.4 \%$ of 114 general autopsy populations were found to have ICAS with $>50 \%$ luminal stenosis (16). Notably, the Chinese Intracranial Atherosclerosis study reported that the prevalence of ICAS was 46.6\% (1335/2864 stroke patients) and concluded that ICAS was the most common vascular lesion of stroke patients in China (17). In an American study, symptomatic ICAS accounted for $9 \%, 15 \%$, and $17 \%$ of all cases of first ischemic strokes in White, Hispanic, and Black patient populations, respectively (18). Most of the epidemiologic studies conducted to date have dealt with the prevalence of ICAS in the general population or stroke patient population without confirmed large vessel occlusion. There have been very few studies reporting on the incidence of underlying ICAS in patients who presented with ELVO. Several retrospective case studies reported that the incidence of severe underlying ICAS ranged from 15.2$30.3 \%$ in patients receiving endovascular thrombectomy for ELV0 treatment (19-21).

Differentiating between ICAS- and embolism-related occlusions is important when performing EVT because the EVT strategy used for ICAS-related occlusions is different from that used for embolic occlusions. This strategy may include the use of a different front-line thrombectomy technique and require rescue treatments in addition to thrombectomy. However, it is generally difficult to differentiate the two types of ELVO based on pre-treatment baseline angiography (18). Thus, understanding the different clinical characteristics of the two types of ELVO is important. Until now, published data specifically focused on the demographics and clinical characteristics of patients with ELVO due to underlying ICAS have been insufficient $(15,19-21)$.

In a retrospective single-center study including patients with middle cerebral artery (MCA) M1 occlusions, ELV0 with underlying ICAS was more frequent in male patients; however, the statistical significance was only marginal (67.5\% vs. $51.2 \%, p=0.072$ ) (15). In another comparative study, there were more men with ICAS- than embolismrelated occlusions ( $91.7 \%$ vs. $53.7 \%, p<0.001)$ (19). Baseline stroke severity is also different between patients with ICAS- and embolism-related ELVO. In a retrospective study that investigated the role of tirofiban in treating patients with ELVO due to ICAS, the baseline National Institute of Health Stroke Scale (NIHSS) score was significantly lower in the ICAS group compared with the non-ICAS group (mean, 14.6 vs. $17.9, p<0.001$ ) (21). Yoon et al. (20) also found that the baseline NIHSS score was significantly lower in the ICAS group compared with the control group (median, 10 vs. $12, p=0.002$ ). Such a trend was also observed in patients with posterior circulation stroke. Kim et al. (22) showed that the initial median NIHSS score tended to be lower in patients with ELVO and ICAS compared with those with an embolism-related stroke (14 vs. $22, p=0.096)$. Lower infarct severity in ELV0 patients with underlying ICAS might be explained by preexisting sufficient collateral circulation in these patients. Kim et al. (23) reported that patients with ICAS had better collateral flow than those with other stroke subtypes, presumably because ICAS requires a longer time for complete arterial occlusion, which allows for the development of adequate collateral flow before the onset of acute stroke. Similarly, another study showed that intracranial large artery atherosclerotic stroke had a much higher mismatch ratio $(p=0.003)$, lower severity of perfusion defect $(p=0.001)$, and higher likelihood of better collaterals $(p<0.001)(24)$.

Vascular risk factors were also different between ELV0 with ICAS and other stroke subtypes. Current smoking was more frequent in patients with MCA occlusions due to ICAS than in those without ICAS (57.5\% vs. $26.8 \%, p$ $<0.001$ ) (15). Lee et al. (19) also reported that smoking was more closely related to ICAS- than embolism-related occlusions ( $50 \%$ vs. $13.4 \%, p<0.001$ ). Diabetes mellitus and hyperlipidemia were other possible risk factors for ICAS-related ELVO. In a study of 140 patients with ELVO, diabetes mellitus and dyslipidemia were more frequent in 
patients with ICAS than in those without ICAS (diabetes mellitus, $45 \%$ vs. $18.9 \%, p=0.001$; and dyslipidemia, $50 \%$ vs. $25.8 \%, p=0.004$ ) (20). In another study, high total cholesterol levels were independently associated with ICAS-related ELVO (odds ratio, 1.019 per $1 \mathrm{mg} / \mathrm{dL}$ of total cholesterol level, $p=0.008$ ) (19). However, in two separate studies, the prevalence of diabetes mellitus did not differ between patients with and without ICAS-related stroke $(15,19)$. Thus, the relationship between diabetes mellitus and ICAS is still a matter of controversy because this relationship can be partly mediated by concomitant hypertension and hyperlipidemia (25).

\section{Pathologic Basis of ELVO Due to Underlying ICAS}

Atherosclerosis is a chronic inflammatory disease characterized by the accumulation of lipids and fibrous tissues in the arterial wall. The "Response to Injury Theory" is now widely accepted to explain the pathogenesis of atherosclerosis (26). The earliest events in atherosclerosis can be triggered by several insults, including physical injury as a result of high blood pressure, turbulent blood flow, hyperlipidemia, or hyperglycemia. Atherosclerosis proceeds from endothelial dysfunction to the formation of a lipid layer or fatty streak within the intima. Subsequently, leukocytes and smooth muscle cells migrate into the vessel wall, and ultimately, degradation of the extracellular matrix and plaque formation occur $(27,28)$. The most common intracranial arteries involved are the proximal and middle segments of the MCAs followed by the basilar artery, supraclinoid internal carotid arteries, and intracranial vertebral arteries $(29,30)$.

There are several possible mechanisms of stroke in patients with ICAS, which include in situ thrombotic occlusion, artery-to-artery embolism, hemodynamic compromise, and branch vessel occlusion. Of these mechanisms, ELVO due to underlying ICAS is mainly caused by in situ thrombosis at the stenotic site (31). When inflammation occurs near an unstable atherosclerotic plaque, it can promote physical disruption of the plaque by superficial erosion, neo-vessel disruption, or fracturing its fibrous elements. Inflammation may promote sudden atheromatous lesion expansion and trigger luminal thrombosis by aggregating platelets, and ultimately, occluding the vessel (32). These findings agree with a postmortem study on MCA plaques. In that study, the authors performed a histologic comparison and found that the degree of luminal stenosis, percentage of plaques containing $>40 \%$ lipid area, and prevalence of intraplaque hemorrhage, neo-vasculature, and thrombus were higher in plaques associated with MCA infarctions compared with those without infarctions (33).

On this pathologic basis, ELVO due to underlying ICAS has a few distinctive features compared with embolism-related occlusions. First, it tends to have a smaller clot burden. Yoon et al. (20) reported on the detailed findings of EVT procedures in patients with ELVO due to underlying ICAS. Of the 29 patients who showed an occlusion with ICAS on the initial angiography and underwent stent-retriever thrombectomy (SRT), only 15 patients showed visible thrombi that were captured on the retrieved stent. The remaining 14 (48.3\%) patients had no captured thrombi despite multiple stent-retriever passages and follow-up angiography in these patients showed severe underlying ICAS. These findings suggested the possibility that very small thrombi might be lost during stent retrieval. Another investigation compared the clot burden scores on gradient echo (GRE) magnetic resonance imaging between ICAS- and embolism-related occlusions (34). In that study, the clot burden score was higher in ICAS- compared with embolismrelated occlusions (median clot burden score on GRE, 8 vs. $6, p=0.009$ ). In the analysis of that study, higher scores indicated smaller clot burdens. These findings suggest that, during EVT procedures, ICAS-related occlusions may be suspected when the clot burden is small. However, a tendency toward smaller clot burdens with ICAS-related occlusions is not directly related to a lower chance of a GRE susceptibility vessel sign. In the past, the GRE susceptibility vessel sign has been regarded to be more associated with embolism-related stroke (35). However, a more recent study reported a high frequency of GRE susceptibility vessel signs $(60.5 \%)$ in patients with ICAS-related occlusions, potentially due to in situ thrombosis (36). This finding was consistent with another study that showed that GRE susceptibility vessel sign frequency was not significantly different between ICAS- and embolism-related occlusions (71.4\% vs. $84.1 \%, p=0.239$ ) (34).

The second distinctive feature of ELVO due to underlying ICAS is a strong tendency for reocclusion or flow stagnation after achieving initial reperfusion with endovascular thrombectomy $(21,31,37)$. Reocclusion may result from subsequent platelet aggregation at the injured endothelium of the occlusion site if the ruptured atherosclerotic plaque remained unchanged after successful removal of the 
intraluminal thrombus. Accordingly, when ICAS-related occlusion is suspected during an endovascular procedure, a repeat angiography 5-10 minutes after the initial reperfusion is needed to observe whether reocclusion occurs $(31,38)$. If reocclusion occurs, additional rescue treatment is usually required to stabilize the irritable endothelium. Reocclusion is a major cause of failed or complicated EVT procedures in patients with ELVO and underlying ICAS (14). Such procedural difficulty may lead to poor functional recovery in these patients. Kim et al. (22) reported that an ICAS-related occlusion was an independent predictor of poor functional outcomes in patients with acute vertebrobasilar occlusions who were treated with EVT. These authors suggested that poor outcomes in ICAS-related occlusion cases could be attributed to longer procedure durations and complicated procedures due to frequent reocclusions. These observations are in agreement with the notion that achieving effective and successful reperfusion is one of the most important determinants of functional outcomes in patients with ELVO undergoing $\operatorname{EVT}(39,40)$. In the following sections, we discuss an endovascular recanalization strategy for patients with ELVO due to underlying ICAS.

\section{Front-Line Thrombectomy Technique for ELVO Due to Underlying ICAS: Stent Retriever versus Contact Aspiration}

Patients with ICAS-related occlusions require a different

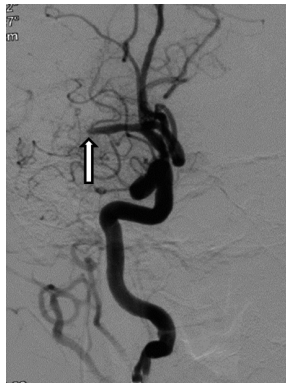

A

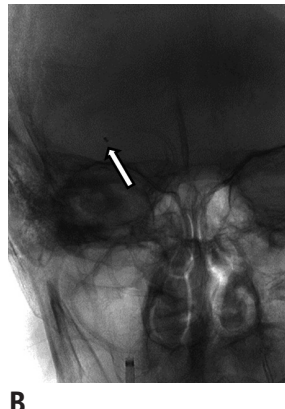

B

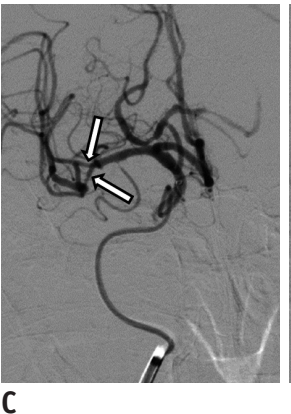

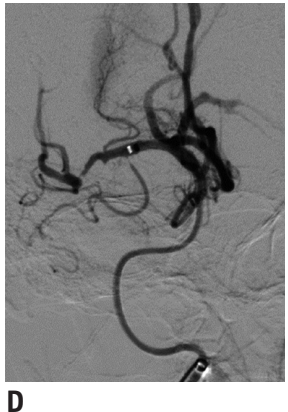

$\mathbf{E}$

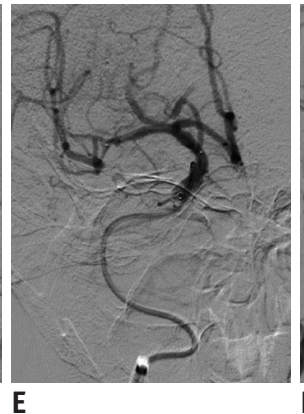

IA tirofiban infusion

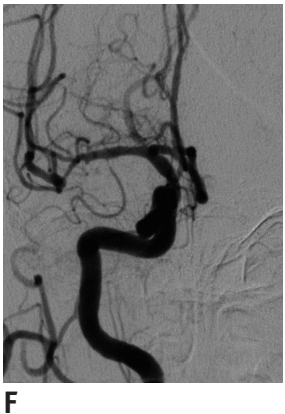

Contact aspiration
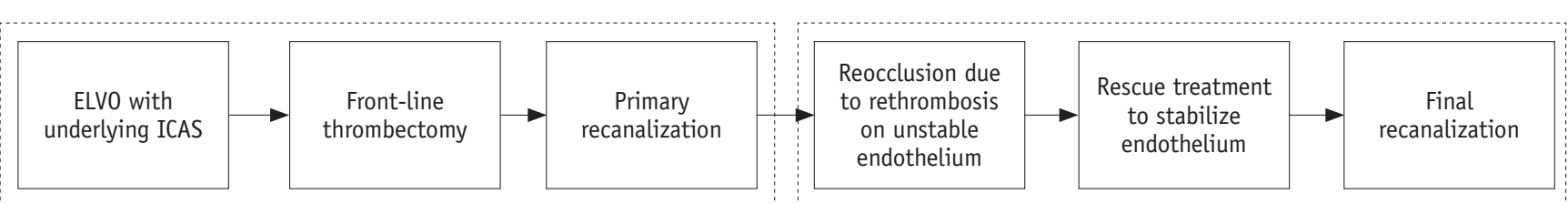

Stent retriever

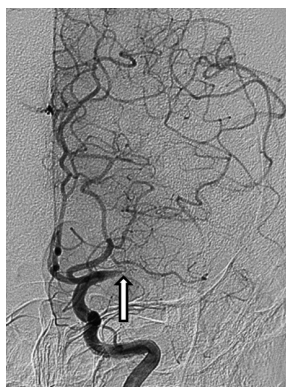

A

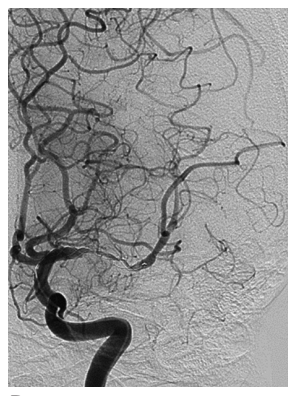

B

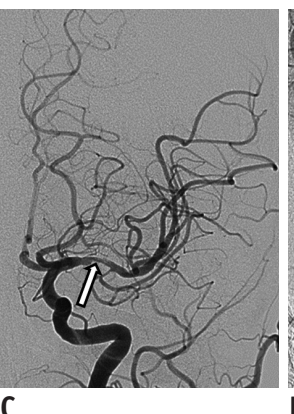

C

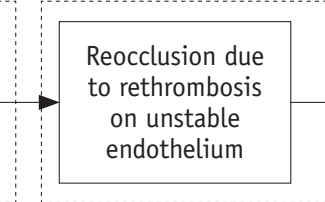

Emergent angioplasty/stenting

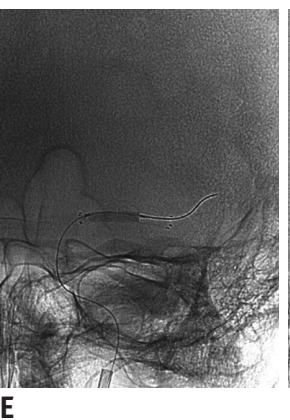

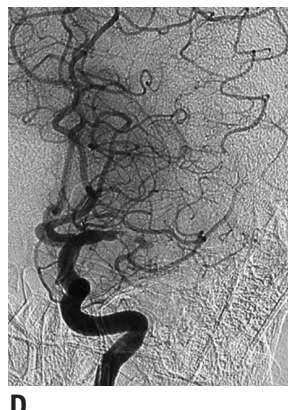

D
$\mathbf{E}$

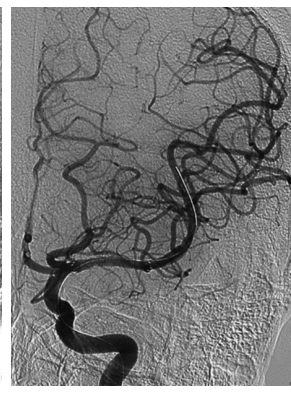

Fig. 1. Therapeutic algorithm for ELVO due to underlying ICAS.

Upper row: A. Baseline angiography shows occlusion (arrow) at distal M1 segment of right MCA. B. Contact aspiration thrombectomy using largebore aspiration catheter. Arrow indicates tip of aspiration catheter. C. Primary reperfusion. Arrows indicate underlying atherosclerotic stenosis. D. Reocclusion and flow stagnation due to rethrombosis on irritable endothelium. E. IA infusion of tirofiban (0.5 mg) over 10 minutes. F. Final reperfusion. Lower row: A. Baseline angiography shows occlusion (arrow) at proximal M1 segment of left MCA. B. Stent-retriever thrombectomy. C. Primary reperfusion. Arrow indicates underlying atherosclerotic stenosis. D. Reocclusion and flow stagnation. E. Emergent angioplasty with stenting. F. Final reperfusion. ELVO = emergent large vessel occlusion, $I A=$ intraarterial, ICAS = intracranial atherosclerotic stenosis, $M C A=$ middle cerebral artery 
EVT strategy from that used for patients with embolic stroke. The treatment strategy may consist of the following two components (Fig. 1): 1) front-line thrombectomy to achieve primary recanalization of the target artery and 2) rescue treatment to stabilize an irritable endothelium on the ICAS segment to prevent reocclusion $(20,21,31)$. Currently, there are two major thrombectomy techniques for treating ELVO, SRT and contact aspiration thrombectomy (CAT). Although several studies have shown comparable efficacy between these two techniques in patients with ELVO (41-44), it remains unclear as to which EVT technique is more effective as a front-line thrombectomy in patients with ELVO due to underlying ICAS. To date, data from only a few small, single-arm case series are available regarding the efficacy of SRT or CAT as a front-line thrombectomy for ELVO with underlying severe ICAS. Kang et al. (21) reported that front-line CAT was effective in $62.5 \%$ (25/40) of patients with in situ thrombo-occlusion, and conversion to SRT was required for the remaining $37.5 \%$ of cases. In contrast, two separate front-line SRT studies for ICAS-related occlusions demonstrated that SRT was effective in $88.9 \%$ (8 of 9) and $93.1 \%$ (27 of 29) of cases as a front-line thrombectomy, even though additional rescue treatment was required in the majority of the cases ( $77.8 \%$ and $95 \%$, respectively) (20, 37). Recently, we conducted a multicenter retrospective analysis to compare the procedural characteristics and outcomes between front-line SRT and CAT in 130 patients with ELVO due to underlying ICAS (unpublished data). We found that SRT was associated with a shorter time from puncture to initial reperfusion (17 vs. $31 \mathrm{~min}$ ), shorter procedure duration ( 39 vs. $75.5 \mathrm{~min}$ ), and lower rate of switching to an alternative thrombectomy technique ( $4.3 \%$ vs. $40 \%$ ) compared with CAT. Thus, SRT may be more suitable than CAT as a front-line thrombectomy technique in patients with ELVO due to ICAS.

There are several possible explanations for the superiority of SRT over CAT as a front-line thrombectomy achieving primary reperfusion. First, a contact problem may exist with the CAT technique. Because ICAS-related occlusions tend to have a smaller clot burden than embolism-related occlusions, clot aspiration is not very difficult when the contact is appropriate between the aspiration catheter tip and thrombus (Fig. 2A, B). However, most ICAS segments are tapered and irregularly shaped due to preexisting atherosclerosis within the vessel wall. Therefore, it may be difficult to place the tip of a large-bore aspiration catheter into contact with the proximal surface of a thrombus (Fig. $2 \mathrm{C}$ ). With the CAT technique, contact is the primary factor for achieving successful recanalization. In contrast, the stent retriever is deployed across the stenotic segment and becomes fully engaged with the entire length of a clot (Fig. 2D). Second, when performing SRT, a temporary bypass can be achieved by placing the stent retriever across the target arterial occlusion site. The temporary restoration of flow may cause thrombus reduction or dissolution via endogenous thrombolysis. In addition, this can provide
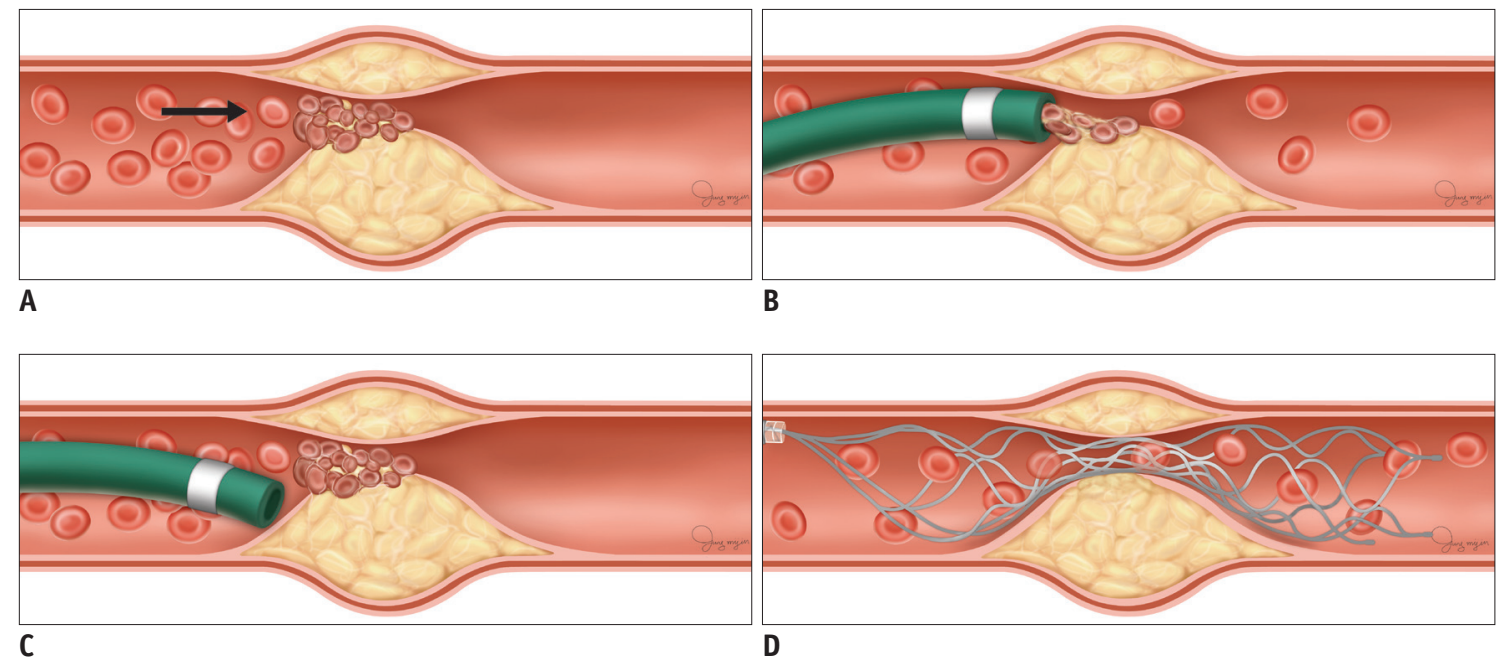

Fig. 2. Illustrations of front-line thrombectomy in EVT for ELVO due to underlying ICAS.

A. In situ thrombosis is one of main pathologies of ELVO due to underlying ICAS. Arrow indicates antegrade blood flow. B. Clot retrieval is not very difficult when contact is appropriate between large-bore aspiration catheter tip and proximal surface of clot. C. At times, it may be difficult to place catheter tip in contact with clot because of tapered and irregular anatomy of stenotic lumen. D. Stent retriever is deployed across stenotic segment and becomes fully engaged with entire length of clot. EVT = endovascular therapy 
another potential advantage by more readily revealing the underlying culprit stenosis after retrieving the stent. Therefore, it is possible to plan a subsequent rescue therapy earlier, which may be helpful for reducing procedure time and achieving successful reperfusion.

\section{Rescue Treatment for Underlying ICAS:} Intraarterial Infusion of an Antiplatelet Agent versus Emergent Angioplasty with or without Stenting

As previously mentioned, instant reocclusion frequently occurs in ELVO with underlying ICAS even when a frontline EVT is successful in achieving primary reperfusion $(21,37)$ (Fig. 3A). In a study on the outcomes of ELVO

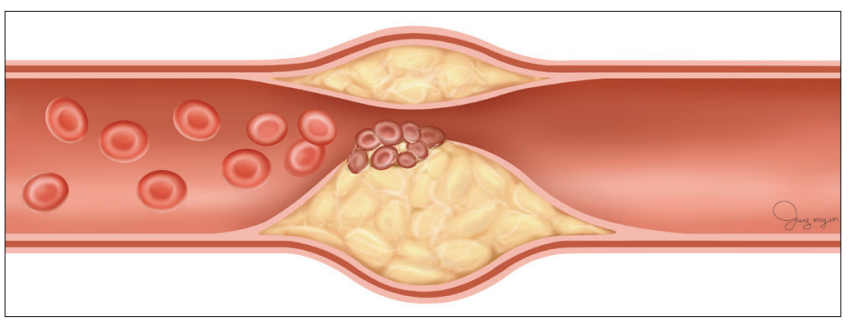

A

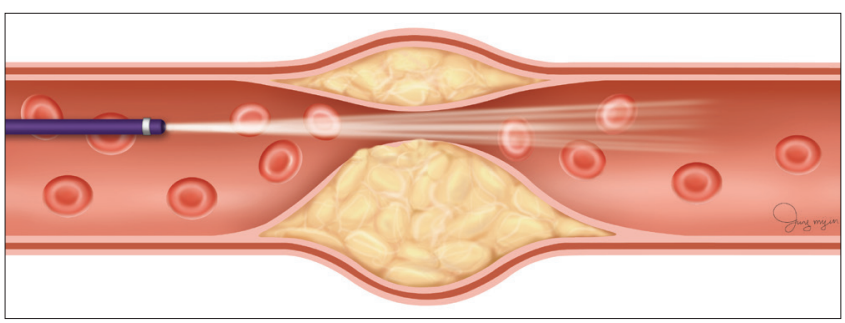

B

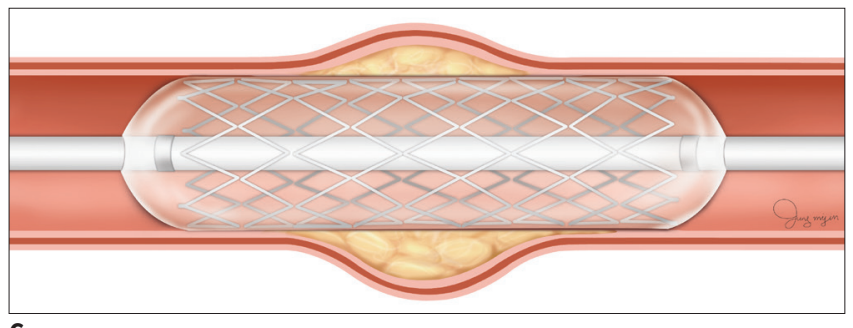

C

Fig. 3. Illustrations of rescue treatment for reocclusion and flow stagnation after primary reperfusion in EVT for ELVO due to underlying ICAS.

A. Although front-line thrombectomy can be successful in achieving primary reperfusion in ICAS-related ELV0, instant reocclusion can frequently occur because remaining endothelium is irritable due to rethrombosis. B. Intraarterial use of glycoprotein IIb/IIIa inhibitors, such as tirofiban, can stabilize irritable endothelium. C. Emergent angioplasty with or without stenting can treat stenosis and is another viable option for preventing reocclusion. with underlying ICAS, the incidence of reocclusion was significantly higher in the ICAS-related occlusion group compared with the embolism-related occlusion group (65\% vs. $3.3 \%, p<0.001)(21)$. Lee et al. (37) also reported that seven of nine patients with ICAS-related occlusions required additional rescue treatment due to reocclusion after primary reperfusion.

Stabilization of an irritable endothelium on the stenotic segment appears to be an important rescue treatment to prevent reocclusion for ELVO with underlying ICAS. However, studies regarding such treatments have been limited. Some case series have reported on outcomes after treatment with an intraarterial antiplatelet agent (glycoprotein IIb/IIIa inhibitor) or intracranial angioplasty with or without stenting to prevent reocclusion $(20,21,45,46)$. Tirofiban is a short-acting glycoprotein IIb/IIIa inhibitor and competitively inhibits platelet aggregation mediated by fibrinogen. Because the major components of clots formed by in situ thrombosis are platelets and fibrin, intraarterial infusion of an antiplatelet drug at the occlusion site may be a reasonable therapeutic option in patients with in situ thrombosis (Fig. 3B) (32). The intraarterial use of tirofiban during EVT procedures has been shown to be safe and effective $(21,47)$. Kang et al. (21) previously reported on the efficacy of intraarterial low-dose tirofiban infusion in patients with ICAS and instant reocclusion following thrombectomy. When reocclusion occurred after primary reperfusion with front-line EVT, the authors intraarterially administered low-dose tirofiban to stabilize the irritable endothelium. of the 35 patients who experienced reocclusion, $85.7 \%(30 / 35)$ had modified treatment in cerebral infarction (m-TICI) $2 / 3$ recanalization and $74.3 \%$ (26/35) had m-TICI $2 b / 3$ recanalization. Rescue stenting was necessary for the remaining five patients. Notably, there were no symptomatic intracranial hemorrhages in this cohort, which might be related to the lower dosage of tirofiban (0.5-1.0 mg), absence of additional systemic tirofiban maintenance after the procedure, and routine checkup with flat-detector $\mathrm{C}$-arm cone-beam computed tomography to exclude intracranial hemorrhage prior to tirofiban infusion (21).

The main disadvantage of intraarterial tirofiban infusion is the development of subacute or late reocclusion of the treated artery due to the underlying severe stenosis itself remaining unresolved $(15,21)$. Recent studies have also suggested that emergent angioplasty with or without stenting can be a viable therapeutic option in patients 
with ELV0 secondary to underlying ICAS (Fig. 3C) $(20,47)$. The possible disadvantages of intracranial angioplasty with or without stenting with hyperacute stroke include adjacent perforator occlusion, vessel injury, and in-stent thrombosis $(30,48)$. Despite these concerns, emergent angioplasty with or without stenting has been shown to be feasible with favorable outcomes. In a United States population-based study, Al Kasab et al. (45) reported that emergent intracranial angioplasty with (32/36 patients) or without (2/36 patients) stenting using the Wingspan stent system (Stryker, Fremont, CA, USA) was safe and had good functional outcomes in patients with ICASrelated occlusions. Although, in that study, the procedure time was significantly longer in the ICAS group compared with the embolism group ( 98.5 vs. $37.1 \mathrm{~min}$ ) due to EVT complexity, $64.7 \%$ of the patients had final successful reperfusion (m-TICI $2 \mathrm{~b}$ or 3 ) and $42.4 \%$ showed good recovery (modified Rankin scale [mRS]: $0-2$ ) at 3 months. Yoon et al. (20) reported on the outcome of the emergent angioplasty with or without stenting rescue strategy using the Wingspan stent system (Stryker) in patients with insufficient reperfusion achieved by SRT. Of the 40 patients with ELVO due to underlying ICAS, 38 patients received intracranial angioplasty with $(n=24)$ or without $(n=14)$ stenting. This rescue strategy was associated with a high rate of good outcomes (62.5\%, mRS: $0-2$ at 3 months) and low rate of hemorrhagic complications $(7.5 \%$, symptomatic hemorrhage). Based on these results, it can be reemphasized that achieving successful reperfusion is one of the most important predictors of good functional recovery and is more important than time for patients with ELVO as well as those with ICAS-related occlusions (40).

We recently published a multicenter study comparing intraarterial tirofiban infusion and intracranial angioplasty with or without stenting in patients with ELVO due to underlying ICAS (49). Our study demonstrated that both of the treatment approaches were comparably safe and effective as rescue treatments for reocclusion. In our study, overall successful reperfusion and good functional outcome were achieved in $95 \%$ and $60 \%$ of patients, respectively. Based on these data, we suggested that physicians might choose the therapeutic option that they are more familiar with (i.e., either intraarterial tirofiban infusion or intracranial angioplasty with or without stenting) as an adjuvant strategy to prevent reocclusion. If one of these methods failed to achieve effective reperfusion, the other could be used as an additional rescue approach. However, each option has advantages and disadvantages, such as elevating hemorrhagic concerns with tirofiban infusion and the chance of rethrombosis with angioplasty with or without stenting. Thus, selection of the safer option based on patient condition (i.e., baseline infarct volume, degree of stenosis, arterial tortuosity, and lesion length) may be advantageous.

\section{Future Perspectives and Conclusions}

Until now, there have been no large clinical trials focused on patients with ELVO due to underlying ICAS, and the reported data are mostly based on retrospective case series. There are several practical issues that need to be investigated within the next couple of years. First, the efficacy of detachable stent retrievers (Solitaire; Medtronic, Irvine, CA, USA) for permanent deployment as a rescue for ELVO due to underlying ICAS should be evaluated. Most previous intracranial stenting studies have been performed using a Wingspan stent system. Recently, in cases of failed thrombectomy, researchers have reported a high rate of successful recanalization using a permanent stenting rescue strategy with the Solitaire stent $(50,51)$. The evaluation of the long-term efficacy of such a practice is needed to ensure that the radial forces of the Solitaire stent are sufficient to endure the remaining ICAS. Second, it is still unclear whether any additional endovascular procedures are always required in patients with ICAS-related occlusions, even after adequate reperfusion has been achieved by front-line EVT. Given that these patients have presumably had long-standing stenosis that was not symptomatic until the acute event, it should be investigated whether an EVT followed by the best medical therapy strategy could be an alternative option for patients with ELVO due to underlying ICAS. Third, postprocedural medical treatment is very important for ICAS. The current best medical treatments for ICAS involve strong antiplatelet and statin administration, which leads to stenosis regression more often than expected, even in patients without intervention (52). However, such treatments should be cautiously applied during the immediate postprocedural period for patients with ICAS-related occlusion, especially when EVT follows intravenous thrombolysis.

In summary, ICAS is one of the most common causes of acute ischemic stroke worldwide. Therefore, it is not rare for neurointerventionists to encounter ELVO due to underlying ICAS during EVT procedures. In situ thrombotic occlusion, 
which is the main mechanism of ELVO due to underlying ICAS, is strongly related to inflammatory processes due to unstable atherosclerotic plaques and plaque rupture. Because of its unique pathologic basis, ELVO due to underlying ICAS generally requires two endovascular treatment strategy steps, including front-line thrombectomy to achieve primary recanalization and rescue treatment to stabilize the irritable endothelium on the ICAS segment to prevent reocclusion. Previous case series have suggested that endovascular thrombectomy using modern devices and subsequent rescue therapy, such as intraarterial tirofiban infusion and angioplasty with or without stenting, are also effective and safe for treating patients with ELVO due to severe underlying ICAS. Further randomized studies are needed to establish the optimal endovascular treatment option as well as the best periprocedural management for ELVO due to underlying ICAS.

\section{Conflicts of Interest}

The authors have no potential conflicts of interest to disclose.

\author{
ORCID iDs \\ Woong Yoon \\ https://orcid.org/0000-0002-8598-3127 \\ Dong-Hun Kang \\ https://orcid.org/0000-0002-2977-9340
}

\section{REFERENCES}

1. The National Institute of Neurological Disorders and Stroke rtPA Stroke Study Group. Tissue plasminogen activator for acute ischemic stroke. N Engl J Med 1995;333:1581-1588

2. Hacke W, Kaste M, Bluhmki E, Brozman M, Dávalos A, Guidetti $D$, et al.; ECASS Investigators. Thrombolysis with alteplase 3 to 4.5 hours after acute ischemic stroke. $N$ Engl J Med 2008;359:1317-1329

3. Bhatia R, Hill MD, Shobha N, Menon B, Bal S, Kochar P, et al. Low rates of acute recanalization with intravenous recombinant tissue plasminogen activator in ischemic stroke: real-world experience and a call for action. Stroke 2010;41:2254-2258

4. del Zoppo GJ, Poeck K, Pessin MS, Wolpert SM, Furlan AJ, Ferbert $A$, et al. Recombinant tissue plasminogen activator in acute thrombotic and embolic stroke. Ann Neurol 1992;32:7886

5. Berkhemer OA, Fransen PS, Beumer D, van den Berg LA, Lingsma HF, Yoo AJ, et al.; MR CLEAN Investigators. A randomized trial of intraarterial treatment for acute ischemic stroke. N Engl J Med 2015;372:11-20

6. Campbell BC, Mitchell PJ, Kleinig TJ, Dewey HM, Churilov L, Yassi $\mathrm{N}$, et al.; EXTEND-IA Investigators. Endovascular therapy for ischemic stroke with perfusion-imaging selection. $N$ Engl J Med 2015;372:1009-1018

7. Goyal M, Demchuk AM, Menon BK, Eesa M, Rempel JL, Thornton J, et al.; ESCAPE Trial Investigators. Randomized assessment of rapid endovascular treatment of ischemic stroke. N Engl J Med 2015;372:1019-1030

8. Jovin TG, Chamorro A, Cobo E, de Miquel MA, Molina CA, Rovira A, et al.; REVASCAT Trial Investigators. Thrombectomy within 8 hours after symptom onset in ischemic stroke. N Engl J Med 2015;372:2296-2306

9. Kim YD, Cha MJ, Kim J, Lee DH, Lee HS, Nam CM, et al. Increases in cerebral atherosclerosis according to CHADS2 scores in patients with stroke with nonvalvular atrial fibrillation. Stroke 2011;42:930-934

10. Bang OY. Intracranial atherosclerosis: current understanding and perspectives. J Stroke 2014;16:27-35

11. Gorelick PB, Wong KS, Bae HJ, Pandey DK. Large artery intracranial occlusive disease: a large worldwide burden but a relatively neglected frontier. Stroke 2008;39:2396-2399

12. Libby P. Inflammation in atherosclerosis. Nature 2002;420:868-874

13. Kim JS, Bonovich D. Research on intracranial atherosclerosis from the East and West: why are the results different? J Stroke 2014;16:105-113

14. Kim BM. Causes and solutions of endovascular treatment failure. J Stroke 2017;19:131-142

15. Hwang YH, Kim YW, Kang DH, Kim YS, Liebeskind DS. Impact of target arterial residual stenosis on outcome after endovascular revascularization. Stroke 2016;47:1850-1857

16. Leung SY, Ng TH, Yuen ST, Lauder IJ, Ho FC. Pattern of cerebral atherosclerosis in Hong Kong Chinese. Severity in intracranial and extracranial vessels. Stroke 1993;24:779-786

17. Wang Y, Zhao X, Liu L, Soo YO, Pu Y, Pan Y, et al.; CICAS Study Group. Prevalence and outcomes of symptomatic intracranial large artery stenoses and occlusions in China: the Chinese Intracranial Atherosclerosis (CICAS) study. Stroke 2014;45:663-669

18. White H, Boden-Albala B, Wang C, Elkind MS, Rundek T, Wright $C B$, et al. Ischemic stroke subtype incidence among Whites, Blacks, and Hispanics: the Northern Manhattan Study. Circulation 2005;111:1327-1331

19. Lee JS, Hong JM, Lee KS, Suh HI, Demchuk AM, Hwang YH, et al. Endovascular therapy of cerebral arterial occlusions: intracranial atherosclerosis versus embolism. J Stroke Cerebrovasc Dis 2015;24:2074-2080

20. Yoon W, Kim SK, Park MS, Kim BC, Kang HK. Endovascular treatment and the outcomes of atherosclerotic intracranial stenosis in patients with hyperacute stroke. Neurosurgery 2015;76:680-686; discussion 686

21. Kang DH, Kim YW, Hwang YH, Park SP, Kim YS, Baik SK. Instant reocclusion following mechanical thrombectomy of in 
situ thromboocclusion and the role of low-dose intra-arterial tirofiban. Cerebrovasc Dis 2014;37:350-355

22. Kim YW, Hong JM, Park DG, Choi JW, Kang DH, Kim YS, et al. Effect of intracranial atherosclerotic disease on endovascular treatment for patients with acute vertebrobasilar occlusion. AJNR Am J Neuroradiol 2016;37:2072-2078

23. Kim SJ, Ryoo S, Kim GM, Chung CS, Lee KH, Bang OY. Clinical and radiological outcomes after intracranial atherosclerotic stroke: a comprehensive approach comparing stroke subtypes. Cerebrovasc Dis 2011;31:427-434

24. Kim SJ, Seok JM, Bang OY, Kim GM, Kim KH, Jeon P, et al. MR mismatch profiles in patients with intracranial atherosclerotic stroke: a comprehensive approach comparing stroke subtypes. J Cereb Blood Flow Metab 2009;29:1138-1145

25. Turan TN, Cotsonis G, Lynn MJ, Chaturvedi S, Chimowitz M; Warfarin-Aspirin Symptomatic Intracranial Disease (WASID) Trial Investigators. Relationship between blood pressure and stroke recurrence in patients with intracranial arterial stenosis. Circulation 2007;115:2969-2975

26. Ross R. Cell biology of atherosclerosis. Annu Rev Physiol 1995; 57:791-804

27. Velican C, Velican D. Atherosclerotic involvement of human intracranial arteries with special reference to intimal necrosis. Atherosclerosis 1982;43:59-69

28. Velican C, Anghelescu M, Velican D. Preliminary study on the natural history of cerebral atherosclerosis. Med Interne 1981;19:137-145

29. Chimowitz MI, Lynn MJ, Howlett-Smith H, Stern BJ, Hertzberg VS, Frankel MR, et al.; Warfarin-Aspirin Symptomatic Intracranial Disease Trial Investigators. Comparison of warfarin and aspirin for symptomatic intracranial arterial stenosis. N Engl J Med 2005;352:1305-1316

30. Chimowitz MI, Lynn MJ, Derdeyn CP, Turan TN, Fiorella D, Lane BF, et al.; SAMMPRIS Trial Investigators. Stenting versus aggressive medical therapy for intracranial arterial stenosis. $N$ Engl J Med 2011;365:993-1003

31. Lee JS, Hong JM, Kim JS. Diagnostic and therapeutic strategies for acute intracranial atherosclerosis-related occlusions. J Stroke 2017;19:143-151

32. Kim SK, Yoon W, Kim TS, Kim HS, Heo TW, Park MS. Histologic analysis of retrieved clots in acute ischemic stroke: correlation with stroke etiology and gradient-echo MRI. AJNR Am J Neuroradiol 2015;36:1756-1762

33. Chen XY, Wong KS, Lam WW, Zhao HL, Ng HK. Middle cerebral artery atherosclerosis: histological comparison between plaques associated with and not associated with infarct in a postmortem study. Cerebrovasc Dis 2008;25:74-80

34. Suh HI, Hong JM, Lee KS, Han M, Choi JW, Kim JS, et al. Imaging predictors for atherosclerosis-related intracranial large artery occlusions in acute anterior circulation stroke. $J$ Stroke 2016;18:352-354

35. Cho KH, Kim JS, Kwon SU, Cho AH, Kang DW. Significance of susceptibility vessel sign on $\mathrm{T}^{*}$-weighted gradient echo imaging for identification of stroke subtypes. Stroke
$2005 ; 36: 2379-2383$

36. Cho KH, Yoon Y, Sohn SI, Kim JS. Susceptibility vessel signs on $\mathrm{T}^{*}$-weighted gradient echo images in patients with cerebral atherosclerosis. Int J Stroke 2014;9:E32

37. Lee JS, Hong JM, Lee KS, Suh HI, Choi JW, Kim SY. Primary stent retrieval for acute intracranial large artery occlusion due to atherosclerotic disease. J Stroke 2016;18:96-101

38. Baek JH, Kim BM, Kim DJ, Heo JH, Nam HS, Song D, et al. Importance of truncal-type occlusion in stentriever-based thrombectomy for acute stroke. Neurology 2016;87:1542-1550

39. Manning NW, Chapot R, Meyers PM. Endovascular stroke management: key elements of success. Cerebrovasc Dis 2016;42:170-177

40. Yoon W, Kim SK, Park MS, Baek BH, Lee YY. Predictive factors for good outcome and mortality after stent-retriever thrombectomy in patients with acute anterior circulation stroke. J Stroke 2017;19:97-103

41. Lapergue B, Blanc R, Gory B, Labreuche J, Duhamel A, Marnat $G$, et al.; ASTER Trial Investigators. Effect of endovascular contact aspiration vs stent retriever on revascularization in patients with acute ischemic stroke and large vessel occlusion: the ASTER randomized clinical trial. JAMA 2017;318:443-452

42. Kang DH, Park J. Endovascular stroke therapy focused on stent retriever thrombectomy and direct clot aspiration: historical review and modern application. J Korean Neurosurg Soc 2017;60:335-347

43. Gory B, Mazighi M, Blanc R, Labreuche J, Piotin M, Turjman F, et al. Mechanical thrombectomy in basilar artery occlusion: influence of reperfusion on clinical outcome and impact of the first-line strategy (ADAPT vs stent retriever). J Neurosurg 2018;129:1482-1491

44. Stapleton CJ, Leslie-Mazwi TM, Torok CM, Hakimelahi R, Hirsch JA, Yoo AJ, et al. A direct aspiration first-pass technique vs stentriever thrombectomy in emergent large vessel intracranial occlusions. J Neurosurg 2018;128:567-574

45. Al Kasab S, Almadidy Z, Spiotta AM, Turk AS, Chaudry MI, Hungerford JP, et al. Endovascular treatment for AIS with underlying ICAD. J Neurointerv Surg 2017;9:948-951

46. Lee YY, Yoon W, Kim SK, Baek BH, Kim GS, Kim JT, et al. Acute basilar artery occlusion: differences in characteristics and outcomes after endovascular therapy between patients with and without underlying severe atherosclerotic stenosis. AJNR Am J Neuroradiol 2017;38:1600-1604

47. Baik SK, Oh SJ, Park KP, Lee JH. Intra-arterial tirofiban infusion for partial recanalization with stagnant flow in hyperacute cerebral ischemic stroke. Interv Neuroradiol 2011;17:442-451

48. Fiorella D, Derdeyn CP, Lynn MJ, Barnwell SL, Hoh BL, Levy EI, et al.; SAMMPRIS Trial Investigators. Detailed analysis of periprocedural strokes in patients undergoing intracranial stenting in Stenting and Aggressive Medical Management for Preventing Recurrent Stroke in Intracranial Stenosis (SAMMPRIS). Stroke 2012;43:2682-2688 
49. Kang DH, Yoon W, Kim SK, Baek BH, Lee YY, Kim YW, et al. Endovascular treatment for emergent large vessel occlusion due to severe intracranial atherosclerotic stenosis. J

Neurosurg 2018 Jun 1 [Epub ahead of print]. https://doi. org/10.3171/2018.1.JNS172350

50. Baek JH, Kim BM, Kim DJ, Heo JH, Nam HS, Yoo J.

Stenting as a rescue treatment after failure of mechanical thrombectomy for anterior circulation large artery occlusion.
Stroke 2016;47:2360-2363

51. Chang Y, Kim BM, Bang OY, Baek JH, Heo JH, Nam HS, et al. Rescue stenting for failed mechanical thrombectomy in acute ischemic stroke: a multicenter experience. Stroke 2018;49:958-964

52. Leung TW, Wang L, Soo YOY, Ip VHL, Chan AYY, Au LWC, et al. Evolution of intracranial atherosclerotic disease under modern medical therapy. Ann Neurol 2015;77:478-486 